\title{
Las "tomas" de tierras y el problema de la vivienda en Latinoamérica
}

\section{Antonio Melo*}

\section{Resumen}

Se trata de un trabajo que recoge de forma detallada las muy diversas realidades bajo las que se produce y materializa el complejo fenómeno de las "tomas" de tierras en Latinoamérica. El libro comienza con una recopilación de textos de Nora Clichevsky, Edésio Fernandes y Mike Davis que componen las bases conceptuales de este informe. Se ofrece una visión del presente de las "tomas" a través del análisis de los datos obtenidos mediante una encuesta de veinte preguntas, realizada a diferentes organismos pertenecientes a 18 países latinoamericanos; así como mediante la inclusión de tres casos de estudio: el programa de "ocupación guiada" de Alto Trujillo (Perú), la mejora "caso a caso y casa a casa" de cuatrocientas viviendas precarias en Ciudad Sandino (Nicaragua) y la "toma" y desalojo del "Campamento Peñalolén" en Santiago de Chile. El informe concluye con una serie de comentarios, deducciones, conclusiones y tres anexos con información pormenorizada sobre el tema en estudio: bibliografía, vocabulario específico, valores sobre la situación de la población tugurizada y centros de análisis sobre asentamientos informales.

\section{Palabras clave}

Toma de Tierras; Asentamientos informales; Desalojos; Regularización; Consolidación urbana.

\section{Abstract: Land "seizures" and the housing problem in Latin America}

This work sets out in detail the very diverse realities under which it produces and embodies the complex phenomenon of land "seizures" in Latin America. The book begins with a collection of texts by Nora Clichevsky, Edesio Fernandes and Mike Davis which makes up the conceptual basis of this report. A current vision of "seizures" is offered through analysis of data obtained by means of a 20-question survey, conducted in different organizations belonging to 18 Latin American countries; as well as by the inclusion of three case studies: the program of "guided occupation" of Alto Trujillo (Peru), the improvement "case by case, house by house" of four hundred shacks in Ciudad Sandino (Nicaragua) and the "seizure" and the eviction of "Peñalolén Camp" in Santiago de Chile. The report concludes with a series of comments, deductions, conclusions and three appendices with detailed information on the subject of study: bibligraphy, specific vocabulary, and values on the status of the slum population and centres of analysis on informal settlements.

\section{Key words}

Land Seizures; Informal Settlements; Eviction; Regularization; Urban Consolidation.

\footnotetext{
* Profesor de Construcciones Arquitectónicas II. Datos de contacto: Escuela Técnica Superior de Ingeniería de Edificación, Universidad de Sevilla. Avda. Reina Mercedes, 4A, 41012 Sevilla. E-mail: melo@us.es.
} 
SALAS, Julián (director). Las "tomas" de tierras urbanas en Latinoamérica hoy ¿problema o solución? Madrid: Secretaría General Técnica del Ministerio de Vivienda, España, Centro de Publicaciones, 2010. $246 p$.

Nos encontramos ante un informe encargado por el Ministerio de Vivienda español a la Cátedra UNESCO de Habitabilidad Básica en la Universidad Politécnica de Madrid (ETSAM). Dicho informe es el resultado de un intenso trabajo en equipo dirigido por el Dr. Julián Salas Serrano, investigador experto en cooperación y gran conocedor de la realidad latinoamericana (ha sido Coordinador Internacional del Subprograma CYTED "Tecnologías para viviendas de interés social en Latinoamérica"). También cabe destacar que es autor de cinco libros, entre ellos, Contra el Hambre de Vivienda: soluciones tecnológicas latinoamericanas (Bogotá: Escala, 1993) y La Industrialización Posible de la Vivienda en Latinoamérica (Bogotá: Escala, 2000), así como de más de un centenar de artículos y trabajos científicos.

Este trabajo recoge de forma diferenciada las muy diversas realidades bajo las que se produce y materializa el complejo fenómeno de las "tomas" de tierras en Latinoamérica. Se estructura en cuatro capítulos: 1. Recopilación de textos sobre el pasado reciente de las "tomas" en Latinoamérica; 2. El presente de las "tomas"; 3 . Tres casos, tres aspectos de "tomas" en Perú, Nicaragua y Chile; 4. Comentarios, deducciones y conclusiones. Al final del informe se incluyen tres anexos: Anexo I, Información complementaria sobre las "tomas" de tierras en América Latina; Anexo II, Glosario de términos y expresiones relacionados con las "tomas"; Anexo III, "Tomas" y tugurios, problemas universales en franco crecimiento.

El libro comienza con un capítulo donde se recopilan los textos que componen las bases conceptuales de este informe. La profesora argentina e in- vestigadora del CONICET Nora Clichevsky aporta su visión sobre el submercado ilegal de la tierra, las formas de acceso al suelo y sobre los aspectos cuantitativos del hábitat popular en América Latina. Del abogado, investigador y urbanista brasileño Edésio Fernandes se reproducen algunos textos sobre la regularización de la tenencia de las tierras tras las "tomas", incluyéndose un interesante cuadro resumen con los aciertos y desaciertos en los procesos de regularización de las "tomas". Del sociólogo y teórico urbano estadounidense Mike Davis, autor de Planeta de Ciudades Miseria (Madrid: Ediciones Akal, 2007), se incluyen algunas partes de dicho libro. El capítulo primero de esta publicación concluye con unos textos extraídos del Informe Mundial sobre Asentamientos Humanos de 2003, The Challenge of Slums (London: Earthscan Publications Ltd., 2003).

En el segundo capítulo se ofrece una visión del presente de las "tomas" a través de la transcripción y análisis de los datos obtenidos mediante una encuesta de 20 preguntas, realizada a diferentes organismos (38 respuestas en total) pertenecientes a 18 países. Cabe destacar la información aportada sobre legislación y programas de ayuda para regularización o consolidación de asentamientos informales. En la encuesta se planteaban siete opciones posibles ante las "tomas": expulsiones, desalojos, tolerancia, reconocimiento, regularización, consolidación e integración urbana de los sectores informales. El informe aporta un interesante análisis de los datos obtenidos: la regularización suma más del $30,52 \%$ de las respuestas y sólo cuatro países (Costa Rica, Ecuador, El Salvador y Venezuela) no la consideran; el $17,87 \%$ corresponde a la opción de la integración urbana de los sectores informales; los desalojos constituyen el $8,42 \%$ de las contestaciones, y en lo referente a las expulsiones es destacable que figura en última posición con un $4,21 \%$. De los datos aportados por las encuestas cabría afirmar que los organismos responsables de los temas de vivien- 
da se inclinan fuertemente y de manera positiva hacia el reconocimiento de la realidad como problema y solución de las "tomas", ya que si se suma regularización, integración y consolidación se llega al $66,26 \%$ del total.

En el capítulo tres se incluye el estudio detallado de tres casos: el programa de "ocupación guiada" de Alto Trujillo (Perú), la mejora "caso a caso y casa a casa" de cuatrocientas viviendas precarias en Ciudad Sandino (Nicaragua) y la "toma" y desalojo del "Campamento Peñalolén" en Santiago de Chile.

El primer caso se ubica en Trujillo, una ciudad de la costa norte de Perú, con una población aproximada de 804.000 habitantes en el área metropolitana. La gran demanda de vivienda por parte de la población con menos recursos está incrementada por la fuerte migración procedente del interior del departamento y desde otras regiones del norte. Esta demanda no puede ser atendida por la débil gestión local, lo que hace que las invasiones de tierras se incrementen. Ante esta situación se desarrolla el Plan de Ocupación Guiada de Alto Trujillo habilitando tierras en este sector situado a unos 7 kilómetros del centro de la ciudad. Se otorga un título de propiedad con la condición de vivir permanentemente en el barrio y cooperar en las obras de consolidación, incluyendo la construcción de la vivienda. Una vez adjudicada la parcela se organiza la población para el acondicionamiento viario y la construcción de letrinas y pozos de agua. A partir de ahí, y dependiendo de la capacidad económica de la familia, se consolida y desarrolla progresivamente la construcción de la vivienda con ayudas institucionales cuando esto es posible. En definitiva, el objetivo prioritario consiste en adelantarse, en la medida de lo posible, a las "tomas" espontáneas excluyendo terrenos no adecuados y reservando el espacio suficiente para los equipamientos.

El segundo ejemplo consiste en la mejora "caso a caso y casa a casa" de cuatrocientas viviendas precarias en Ciudad Sandino (Nicaragua). Esta localidad se ubica en el extremo oeste de la capital de Nicaragua, a unos 12 kilómetros, y contaba en mayo de 2001 con 23.000 familias asentadas. Con este trabajo se busca aportar criterios que permitan realizar de la forma más objetiva posible la selección de beneficiarios para el mejoramiento habitacional mediante donaciones externas. Se parte de la siguiente premisa: la "pobreza es heterogénea" aunque los barrios o tugurios parezcan ser "homogéneamente pobres". Con este enfoque del problema quedan descartadas las soluciones idénticas para cualquier situación por su nula capacidad de respuesta válida y útil a una situación real de pobreza. La metodología para distribuir las ayudas pasó por determinar en primer lugar el estado general del parque habitacional de ciudad Sandino, analizando: el estado físico de las viviendas, el grado medio de hacinamiento, la dotación de servicios de infraestructura y las áreas vulnerables por restricciones físicas (fallas sísmicas, zonas inundables, áreas de actividad volcánica, paso de cables de alta tensión). Se realizó un estudio de la legalidad de las parcelas y se creó un Comité de Selección y Seguimiento del Proyecto. El proceso de selección se efectuó en cuatro fases: difusión e información, valoración de la precariedad, evaluación del tipo "pasa o no pasa" y evaluación técnica personalizada.

El tercer y último caso aportado es la descripción y las enseñanzas de la "toma" y desalojo del Campamento Peñalolén en Santiago de Chile. Puede leerse en el libro que esta toma "realizada el 5 de julio de 1999 por más de 10.000 personas organizadas que ocuparon un terreno de 23,45 has. fue un hecho de gran repercusión, que el autor ha seguido de forma directa desde su inicio, durante el proceso de consolidación del «campamento» (1999-2003): fases de negociación, desalojo y traslado (abril de 2006), hasta su actual transformación en lo que será el «Parque Comunal de Peñalolén», en fase de ejecución (2008)" (Salas, 
2010, p. 190). De este caso se estudia y expone, desde el estado del arte en la materia y el análisis de la toma hasta las fases finales de desalojo, realojo y nuevo uso del terreno. Resultan muy interesantes en el apartado sobre las conclusiones y enseñanzas algunos de los aspectos que destaca el autor: "la celeridad con la que se autodotaron los pobladores de habitabilidad básica", así como el proceso de evolución de "una solución habitacional autoconstruida a una vivienda ejecutada" (Salas, 2010, p. 204). Se puede considerar como inédito en Latinoamérica el proceso que se dio en el desalojo del Campamento Peñalolén, denominado por el autor del informe como la "autodestrucción" por la propia familia autoconstructora, al objetivo de recuperar partes, instalaciones, etc. del cobijo para un posterior segundo uso o una posible venta.

El capítulo cuatro se divide en tres apartados: Comentarios, Deducciones y Conclusiones. Entre los comentarios se encuentra la consideración de que las "tomas" constituyen el inicio de lo que posteriormente serán los asentamientos irregulares, que en Latinoamérica albergan al 31,9\% de la población, con lo que se deduce que es una realidad compleja que forma parte tanto del problema como de la solución. Resulta muy interesante la afirmación que los redactores del informe hacen en el punto 7 de los comentarios: "la historia y desarrollo de las «tomas» de tierras, su situación actual y la evolución de las políticas y programas de los diferentes gobiernos hacia ellas, ponen de manifiesto que la única fuerza capaz de proporcionar alojamiento a los más necesitados, a la escala que es necesaria, está en manos de los propios afectados. La administración pública no tiene, actualmente, capacidad ni recursos para dotar a todos los pobladores necesitados de Latinoamérica de alojamiento digno y de las mínimas condiciones de habitabilidad solamente a base de programas de vivienda. Para conseguirlo, es imprescindible contar con la participación de los necesitados". Otro aspecto reseñable es la constatación de la aparición reciente de todo un conjunto de normativas en relación con los asentamientos precarios y las "tomas" en la práctica totalidad de los países. Se destaca lo variable del porcentaje de zonas tugurizadas en los países estudiados en el presente informe, desde el $80,9 \%$ en Nicaragua hasta el $2 \%$ en Cuba, pasando por el $36,6 \%$ de Brasil o el $8,6 \%$ de Chile. Puede concluirse de este trabajo que ante las "tomas" siempre aparecen tres niveles de actuación: municipal, federal y estatal. Los municipios no suelen contar con atribuciones para regularizar, pero juegan un papel esencial junto a las autoridades federales o estatales en materia de gestión y en los acercamientos a la población. Por último, desde la Cátedra UNESCO y el ICHaB se propone que se preste especial atención a las dos primeras etapas del proceso de "tomas": elección del suelo y parcelación, por ser el germen básico para la posterior regularización o integración urbana de los sectores informales.

El informe concluye, como se ha dicho, con tres anexos. El Anexo I contiene información detallada por países sobre: centros dedicados al estudio de los asentamientos informales, páginas web recomendadas por los autores de las fichas de países, referencias bibliográficas recientes sobre el tema estudiado y reseñas de textos recomendados por los autores del informe. Especialmente interesante, por lo clarificador para quienes no estén familiarizados con el vocabulario que se utiliza, es el Anexo II: "Glosario de términos y expresiones relacionados con las «tomas»". En concreto, si buscamos el término "toma", nos encontramos que no es utilizado con el significado que recoge el Diccionario de la Real Academia Española. En el último Anexo, "«Tomas» y tugurios, problemas universales en franco crecimiento", se incluyen varias tablas con los valores de la población tugurizada a nivel mundial. Se puede ver que el valor aproximado de la inversión necesaria per cápita para mejorar las zonas de vivienda precaria en Lati- 
noamérica y el Caribe en el periodo comprendido entre 2005 y 2020 sería de 1.200 USD Las inversiones de mayor cuantía serían para los capítulos de la construcción de vivienda básica (472 USD) y la provisión de infraestructuras de red (235 USD); para compra y transmisión del suelo se estiman unos 34 USD y por último para planificación y supervisión y para la generación de capacidad 339 USD. Con este último dato se puede deducir la importancia de las primeras etapas de las "tomas" y de la participación de los pobladores en la solución de sus propios problemas.

\section{Cita del artículo}

MELO, Antonio. Las "tomas" de tierras y el problema de la vivienda en Latinoamérica. Hábitat y Sociedad, ํo 1, noviembre de 2010, p. 169-173.

$<$ <ww.habitatysociedad.us.es>.

http://dx.doi.org/10.12795/HabitatySociedad.2010.11.12 